\title{
NOUVELLe
}

\section{La délétion homozygote du gène DPY19L2 est responsable de la majorité des cas de globozoospermie}

Pierre F. Ray, Christophe Arnoult
Équipe génétique, infertilité et thérapeutique, Laboratoire AGIM, CNRS FRE3405,

Université Joseph Fourier de Grenoble, Domaine de la Merci, Faculté de médecine, bâtiment Jean Roget, 38700, La Tronche, France.

pierre.ray@chu-grenoble.fr
> L'infertilité concerne plus de 70 millions de couples dans le monde. II est probable qu'une proportion importante des cas comporte une composante génétique, mais, chez l'homme, peu de gènes ont jusqu'à présent pu être directement liés à des phénotypes d'infertilité masculine. L'identification de gènes agissant directement sur la gamétogenèse permet de mieux comprendre la physiopathologie de l'infertilité et les mécanismes fondamentaux de la reproduction. Notre équipe grenobloise a démontré que le gène DPY19L2 était absent chez une majorité de patients atteints de globozoospermie (présentant des spermatozoïdes ronds et dépourvus d'acrosome) mettant ainsi en évidence l'importance de ce gène dans l'élongation de la tête du spermatozoïde et la formation de l'acrosome [1].

\section{À la recherche du gène muté dans la globozoospermie}

Des centaines de gènes sont nécessaires à la spermatogenèse et l'ovogenèse et cette hétérogénéité génétique freine l'identification de ceux qui sont nécessaires à la fertilité. Deux approches génétiques peuvent être utilisées pour identifier des mutations géniques responsables de pathologies humaines: l'approche gène candidat et l'approche positionnelle par analyse globale du génome. L'approche gène candidat est généralement utilisée comme stratégie diagnostique quand la corrélation génotype-phénotype est établie entre un gène et une pathologie. Cette stratégie peut également être utilisée pour des gènes moins bien connus quand des études réalisées sur un modèle animal, souvent grâce à l'étude de souris knock-out (KO), mettent en évidence un phénotype évocateur d'une pathologie humaine. Bien que de nombreuses souris $K O$ présentent des phénotypes d'infertilité masculine, l'analyse génétique de ces gènes dans des cohortes de patients infertiles n'a généralement donné que des résultats négatifs ou incertains.

Nous avons choisi la stratégie de localisation, grâce à l'analyse par puces à ADN, des polymorphismes nucléotidiques (single nucleotide polymorphisms, SNP) répartis sur tout le génome [1]. Nous avons appliqué cette stratégie à la recherche du gène muté chez neuf patients tunisiens présentant un syndrome rare de globozoospermie. La globozoospermie de type I est caractérisée par la présence dans l'éjaculat de $100 \%$ de spermatozoïdes à tête ronde et dépourvus d'acrosome [2] (Figure 1). L'origine géographique commune de ces patients pouvait laisser penser que certains étaient porteurs d'une même mutation homozygote héritée d'un ancêtre commun. L'analyse des puces à SNP permet de rechercher des régions d'homozygoties communes à plusieurs patients, signature potentielle de la localisation de la mutation responsable de la pathologie [9]. Nous sommes tous porteurs de deux jeux de 22 chromosomes homologues (plus les chromosomes sexuels) hérités de notre père et de notre mère. Ces chromosomes homologues sont quasiment identiques, mais le séquençage du génome a permis d'identifier plusieurs millions de SNP ou nucléotides divergents, les 3 milliards restants étant normalement invariants. Les puces à SNP permettent, grâce à I'hybridation de I'ADN des patients sur un support contenant les séquences de ces SNP, de génotyper jusqu'à un milllion de SNP répartis sur l'ensemble des chromosomes. Elles permettent de constituer un gigantesque code barre du génome des patients. Les données génétiques des patients sont comparées entre elles pour identifier des régions d'homozygoties qui signent la double contribution génétique d'un ancêtre commun aux deux parents du patient testé. Cette homozygotie, qui traduit un appauvrissement de la diversité génétique du patient, favorise la présence de mutations homozygotes

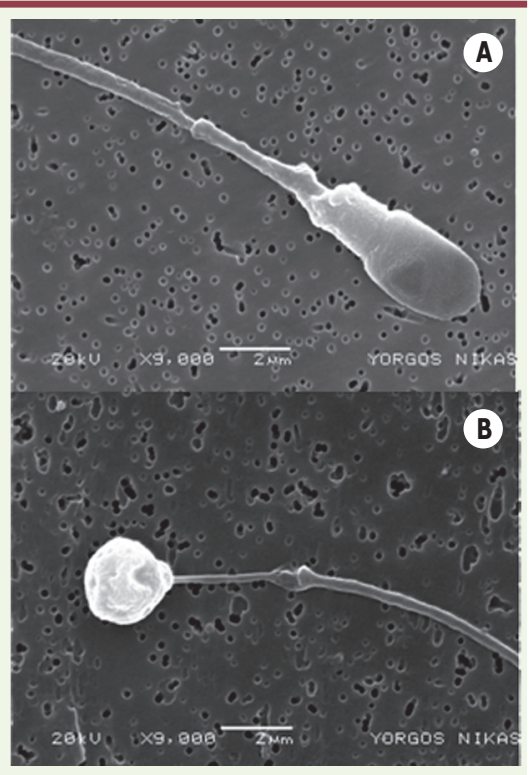

Figure 1. Photographie en microscopie électronique en balayage d'un spermatozoïde normal (A) et d'un spermatozoïde globozoocéphale (B). 


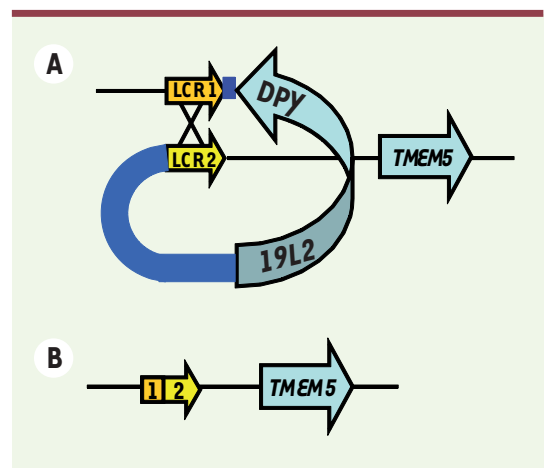

Figure 2. Illustration de la recombinaison par NAHR au locus DPY19L2. Les séquences LCR 1 et 2 s'apparient $(A)$ et donnent un allèle recombiné dépourvu de la séquence située entre les deux LCR contenant uniquement le gène DPY19L2 (B). Le gène le plus proche, TMEM5 (transmembrane protein 5), n'est pas affecté par la recombinaison.

et donc le développement de maladies génétiques. C'est ce que l'on observe dans les populations chez lesquelles le mariage entre individus apparentés est fréquent. La stratégie de recherche de régions d'homozygoties chez des patients issus de familles consanguines ou venant d'isolats génétiques est appelée homozygotie par filiation. Nous avons précédemment utilisé cette stratégie qui avait permis de démontrer que le gène $A U R K C$ (aurora kinase $C$ ) est un acteur majeur de la méiose. En l'absence d'une protéine AURKC fonctionnelle, la méiose était interrompue, les patients produisant des spermatozoïdes macrocéphales tétraploïdes, c'est-à-dire contenant quatre fois la quantité normale d'ADN [3, 4].

\section{Importance de la protéine DPY19L2 pour la formation de l'acrosome}

Dans l'étude des patients atteints de globozoospermie une région d'homozygotie commune à sept des neuf patients a été mise en évidence sur le chromosome 12 [1]. Cette région contient le gène DPY19L2 décrit comme étant exprimé principalement dans les testicules. La fonction de ce gène était inconnue chez I'homme. Une seule étude avait démontré que DPY19, l'ancêtre du gène humain chez le ver Caenorhabditis elegans, était nécessaire à la polarité et à la migration des neuroblastes durant l'embryogenèse. On pouvait donc penser que DPY $19 L 2$ permettrait de spécifier la partie antérieure des spermatides et qu'en son absence l'élongation de la tête ne pourrait avoir lieu. Nous avons tenté de séquencer le gène pour identifier une mutation causale et nous nous sommes aperçu que la totalité du gène était délétée chez sept des patients analysés. Onze patients supplémentaires ont été analysés et huit étaient également porteurs de cette délétion homozygote de la région de DPY19L2. L'analyse de la région entourant DPY $19 L 2$ a permis de mettre en évidence la présence d'une séquence répétée située de part et d'autre du gène d'intérêt. C'est la présence de ces séquences dupliquées (LCR pour low copy repeat) qui est responsable de la survenue de la délétion chez les patients ayant des spermatozoïdes globozoocéphales par un mécanisme de recombinaison homologue non allélique (NAHR) (Figure 2). Le mécanisme de NAHR est connu pour engendrer des délétions ou des duplications entre des séquences répétées localisées à des endroits différents du génome [5]. Ce mécanisme est connu pour contribuer à l'évolution des espèces, en permettant la duplication génique qui peut permettre une spécialisation du gène dupliqué ou au contraire avoir des conséquences délétères en étant responsable de pathologies comme celle que nous avons identifiée [1]. Grâce à l'évolution des technologies et en particulier à l'utilisation accrue de la CGH (comparative genomic hybridization), qui permet de détecter de tels microremaniements, le nombre de pathologies connues attribuables à la NAHR augmente de manière spectaculaire. $0 n$ peut citer le syndrome de Prader Willy, la neurofibromatose de type I ou l'amyotrophie spinale infantile [6].

L'injection intracytoplasmique (ICSI) de spermatozoïdes paraît tout à fait adaptée comme approche de fécondation in vitro chez ces patients ayant des spermatozoïdes globozoocéphales: les spermatozoïdes sont présents, ils ont complété leur méiose et leur absence de capacité de fécondation semble liée à leur incapacité - par absence d'acrosome - à traverser la membrane pellucide de l'ovocyte. De nombreuses tentatives d'ICSI ont été décrites mais les spermatozoïdes de ces patients ont un très faible pouvoir fécondant, responsable d'un taux de réussite très bas [2]. II semblerait que ce défaut soit lié à l'absence secondaire de phospholipase C zêta (PLCzêta) sur le spermatozoïde [7]. Cette protéine est nécessaire à l'initiation des oscillations calciques lors de la fécondation [8]. On peut donc espérer que, pour ces patients, les avancées génétiques seront suivies rapidement par des avancées thérapeutiques, potentiellement grâce à l'utilisation de PLCzêta lors de la fécondation par ICSI. $\diamond$ Homozygous deletion of DPY 19 L2 is responsible for most cases of globozoospermia

\section{CONFLIT D'INTÉRÊTS}

Les auteurs déclarent n'avoir aucun conflit d'intérêts concernant les données publiées dans cet article.

\section{RÉFÉRENCES}

1. Harbuz R, Zouari R, Pierre V, et al. A recurrent deletion of DPY19L2 causes infertility in man by blocking sperm head elongation and acrosome formation. AmJ Hum Genet 2011 ; 88 : 351-61.

2. Dam AH, Feenstra I, Westphal JR, et al.

Globozoospermia revisited. Hum Reprod Update 2007 ; $13: 63-75$.

3. Dieterich K, Soto Rifo R, Faure AK, et al. Homozygous mutation of AURKC yields large-headed polyploid spermatozoa and causes male infertility. Nat Genet 2007 ; 39 : 661-665.

4. Dieterich K, Zouari R, Harbuz R, et al. The Aurora Kinase C c.144delC mutation causes meiosis I arrest in men and is frequent in the North African population. Hum Mol Genet 2009; 18 : 1301-9.

5. Inoue K, Lupski JR. Molecular mechanisms for genomic disorders. Annu Rev Genom Hum Genet 2002 ; 3 : 199-242.

6. Stankiewicz P, Lupski JR. Structural variation in the human genome and its role in disease. Annu Rev Med 2010 ; $61: 437-55$.

7. Heytens $\varepsilon$, Parrington J, Coward K, et al. Reduced amounts and abnormal forms of phospholipase $C$ zeta (PLCzeta) in spermatozoa from infertile men. Hum Reprod 2009; 24 : 2417-28.

8. Yoon SY, Jellerette T, Salicioni AM, et al. Human sperm devoid of PLC, zeta $\mathrm{l}$ fail to induce $\mathrm{Ca}^{2+}$ release and are unable to initiate the first step of embryo development. J Clin Invest 2008 ; 118 : 3671-81.

9. Jordan B. Chroniques génomiques. «Génome personnel » : gadget ou révolution? Med Sci (Paris) $2004 ; 24: 91-4$. 